\title{
Study on superoxide and hydroxyl radicals generated in indirect electrochemical oxidation by chemiluminescence and UV-Visible spectra
}

\author{
ZHANG Bo-Tao ${ }^{1}$, ZHAO Li-Xia ${ }^{1}$, LIN Jin-Ming ${ }^{1,2, *}$ \\ 1. State Key Laboratory of Environmental Chemistry and Ecotoxicology, Research Center for Eco-Environmental Sciences, \\ Chinese Academy of Sciences, Beijing 100085, China. E-mail: zhangbotao@126.com \\ 2. Department of Chemistry, Tsinghua University, Beijing 100084, China
}

Received 24 September 2007; revised 1 November 2007; accepted 29 November 2007

\begin{abstract}
The generation and transformation of radicals on the cathode of indirect electrochemical oxidation were studied by chemiluminescence (CL) and UV-Visible spectra in the reactor with a salt bridge that connected the separated chambers. The CL intensity of $4 \times$ $10^{-9} \mathrm{~mol} / \mathrm{L}$ luminol on the cathode with bubbling oxygen was about seven times that of the intensity without it, which was because of the generation of reactive oxygen species (ROS). The existence of ROS, especially the generation of the superoxide radical, could be affirmed by the fact that the CL intensity of $4 \times 10^{-9} \mathrm{~mol} / \mathrm{L}$ 2-methyl-6-(4-methoxyphenyl)-3,7-dihydroimidazo[1,2-a]pyrazin-3-one with bubbling oxygen was about four times that of the intensity without it. However, there was no chemiluminescence on the anode under the same condition. The change in the UV-Visible spectra of nitro blue tetrazolium and N,N-dimethyl-4-nitrosoaniline at the cathode chamber affirmed the transformation from oxygen to superoxide and hydroxyl radicals. The mechanism of the superoxide and hydroxyl radical generation and transformation on the cathode was discussed with the help of the experimental results and relative references.
\end{abstract}

Key words: chemiluminescence (CL); UV-Visible; radical; indirect electrochemical oxidation

\section{Introduction}

Since the concept of advanced oxidation processes (AOPs) was put forward in the 1980s, a number of literatures have already been devoted to the applications of AOPs to industrial effluents of diverse nature (Glaze et al., 1987; Legrini et al., 1993; Pera-Titus et al., 2004). The AOPs have been defined broadly as those aqueous phase oxidation processes that are based primarily on the intermediacy of reactive species, such as, hydroxyl and superoxide radicals in the mechanism, resulting in the almost complete degradation of the target pollution compound, including a variety of xenobiotics and micropollutions. The production of these radicals of homogenous AOPs is achieved by the combinations of ozone, hydrogen peroxide, and UV radiation or a Fenton reagent. Heterogeneous AOPs mainly include $\mathrm{TiO}_{2}$-mediated photocatalysis and advanced electrochemical oxidation processes (Esplugas $e t$ al., 2002; Andreozzi et al., 1999; Boye et al., 2002).

A radical (also called a "free radical") refers to a molecule with at least one unpaired electron in its outermost shell of electrons. On account of the presence of an unpaired electron, the free radicals are highly reactive

\footnotetext{
* Corresponding author. E-mail: jmlin@mail.tsinghua.edu.cn.
}

to oxidize various organic pollutions. The short half-life and high reactivity of the radicals make it difficult to measure and only a few techniques can be utilized, such as, methods of chemical reaction, spin-trap, and direct detection. Although the spin trap coupled with electron spin resonance (ESR) has been proved to be a credible method for measurement of radicals, it is restricted in sensitivity, inconvenience, and expensive instrument. The methods of chemical reaction, such as, chemiluminescence (CL), UV-Visible (UV-Vis), and fluorescence spectra are popular radical measurements on account of their high sensitivity and availability (Lu et al., 2006; Maeda et al., 2005).

The research group of Nasaka studied the relationship between the properties of $\mathrm{TiO}_{2}$ powder and the photocatalytic productions of $\mathrm{O}_{2}{ }^{-}$and $\mathrm{H}_{2} \mathrm{O}_{2}$ by means of the luminol CL probe method (Hirakawa et al., 2001). Xu et al. (1998) also developed a method for the determination of hydroxyl radicals in the Fenton reaction by chemiluminescence.

Electrochemical methods for recalcitrant toxic waste treatment have attracted a great deal of attention because these methods are environmentally friendly and do not produce new toxic wastes. Electrochemical methods have been successfully applied in the purification of landfill 
leachate, tannery wastes, olive oil wastewaters, textile wastes, and so on (Wu and Zhou, 2001; Panizza and Cerisola, 2004). The electrochemical oxidation process will not be economical because of the high-energy consumption that hinders its large scale application. Since the 1990s, there has been an increasing interest in the indirect oxidation of organic pollutions by reactive species such as hydrogen peroxide, which are generated on the cathode which cut the energy cost dramatically (Shen et al., 2005; Brillas et al., 1995). Although the degradation efficiency of the reactive species has been largely researched and several possible mechanisms of indirect electrochemical oxidation have brought forward, to the authors' knowledge, few of them have adequate and instantaneous evidence about the generation mechanism of the reactive species, especially radicals on the cathode.

In the present work, the generation and transformation of the superoxide and hydroxyl radicals in indirect electrochemical oxidation were investigated with instantaneous and sensitive chemiluminescence and UV-Vis spectra. On the basis of experiment results, the possible mechanisms of radical generation and transformation were put forward and their future applications in pollution degradation were discussed.

\section{Materials and methods}

\subsection{Reagent and materials}

3-Aminophthalhydrazide (luminol) was purchased from Alfa Aesar (Heysham, Great Britain). Nitro blue tetrazolium (NBT) was from Nakalai Tesque Inc. (Kyoto, Japan). N,N-dimethyl-4-nitrosoaniline (RNO) was obtained from Merk \& Co. Inc. (Hohenbrunn, Germany) and 2-methyl-6-(4-methoxyphenyl)-3,7dihydroimidazo[1,2-a]pyrazin-3-one hydrochloride was from the Tokyo Chemical Industry Co. Ltd. (Tokyo, Japan).

All the reagents used in these experiments were of analytical grade or higher, and were used without further purification. Water was purified using a compact ultra pure water system (18.3 M $\Omega / \mathrm{cm}$, Barnstead, Iowa, USA).

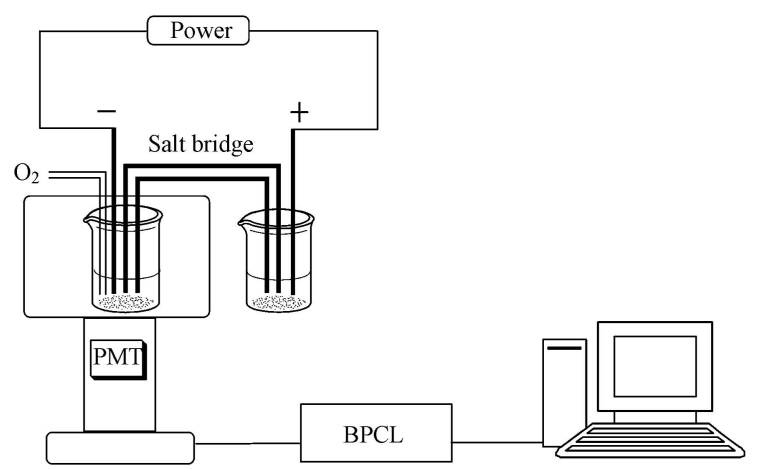

Fig. 1 Schematic diagram of electrolysis and its chemiluminescence detection system. Power: the regulated DC power; cathode: stainless steel; anode: titanium coating with $\mathrm{PbO}_{2}$. Supporting electrolyte: 0.04 $\mathrm{mol} / \mathrm{L} \mathrm{K}_{2} \mathrm{SO}_{4}$. PMT: photomultiplier tube.

\subsection{Electrolysis and chemiluminescence detection sys- tem}

Electrolysis and its CL detection system are shown in Fig.1. The RS1303DF regulated DC power supply (Zefengsheng Instrument Science \& Technology Ltd. Co., Shenzhen, China) with current-voltage monitor was employed to provide electric power. A sheet of stainless steel $304(2 \mathrm{~cm} \times 2 \mathrm{~cm})$ was used as the cathode, whereas, the anode was made of a sheet of titanium $(2 \mathrm{~cm} \times$ $2 \mathrm{~cm}$ ) coating with $\mathrm{PbO}_{2}$ (Zhongke Huiyou Ltd. Co., Shenyang, China). The supporting electrolyte was 0.04 $\mathrm{mol} / \mathrm{L} \mathrm{K}_{2} \mathrm{SO}_{4}$. A $10 \% \mathrm{~K}_{2} \mathrm{SO}_{4}$ salt bridge connected the separated chambers. The voltage of the system was 9.00 $\mathrm{V}$ and the current was about $30 \mathrm{~mA}$. CL reagent $4 \times$ $10^{-9} \mathrm{~mol} / \mathrm{L}$ (luminol or MCLA) was added to cathode and anode compartments separately when it was in the chemiluminescence detector. Oxygen (Hengyuantong Gas Ltd. Co., Beijing, China) was bubbled to the cathode chamber when researching the oxygen effect. The CL signal was collected by a CR-105 photomultiplier tube operated at -920 V (Hamamatsu, Japan). Batch chemiluminescence experiments were carried out in a BPCL luminescence analyzer (Institute of Biophysics, Chinese Academy of Sciences, Beijing, China).

\subsection{UV-Vis absorption spectra measurements}

The electrolysis system was as mentioned earlier. Nitro blue tetrazolium of $10^{-4} \mathrm{~mol} / \mathrm{L}$ or $2 \times 10^{-5} \mathrm{~mol} / \mathrm{L} \mathrm{N}, \mathrm{N}$ dimethyl-4-nitrosoaniline was added to the cathode and anode compartments separately. Oxygen was bubbled to the cathode chamber during the electrolysis process when necessary. Aliquots of the sample were periodically drawn from two chambers and its UV-Vis absorption spectra were measured on the UV-2401 spectrophotometer (Shimadzu, Japan).

\section{Results and discussion}

\subsection{Research radicals by chemiluminescence}

The oxygen reduction reaction (ORR) on the cathode is a multielectron reaction and the following pathways of ORR are generally accepted (Shao et al., 2006). In a direct four-electron reduction, four electrons are transferred:

$\mathrm{O}_{2}+4 \mathrm{H}^{+}+4 \mathrm{e}^{-} \longrightarrow 2 \mathrm{H}_{2} \mathrm{O}$

and a series of pathways that involve $\mathrm{H}_{2} \mathrm{O}_{2}$ as an intermediate:

$\mathrm{O}_{2}+2 \mathrm{H}^{+}+2 \mathrm{e}^{-} \longrightarrow \mathrm{H}_{2} \mathrm{O}_{2}$

$\mathrm{H}_{2} \mathrm{O}_{2}+2 \mathrm{e}^{-} \longrightarrow 2 \mathrm{H}_{2} \mathrm{O}$

Several possible mechanisms of indirect electrochemical oxidation have brought forward that there are possible additional steps and other reactive oxygen intermediates involved in Reactions (2) and (3), which have been used in the indirect oxidation of organic pollution compounds (Brillas et al., 1995; Yu et al., 2006). However, to the authors' knowledge, few of them have adequate or 
instantaneous evidence about the generation mechanism of the reactive species.

$\mathrm{CL}$ is a powerful analytical technique that has excellent sensitivity, a wide linear dynamic range, and requires relatively simple and inexpensive instrumentation. Moreover, chemiluminescence is an effective method in the research of radicals, because there exits a promising combination between the instantaneousness of CL and the short life time of radicals (Lu et al., 2006).

Luminol-amplified CL can offer high sensitivity and it has been widely used for the detection of rapid and complex responses of radicals in different systems. To begin with, ionization luminol can be univalently oxidized by strong oxidants such as hydroxyl radical, to yield a luminol radical, which reacts with the superoxide anion radical to yield an unstable endoperoxide. The transient luminol endoperoxide decomposes into an electronically excited aminophthalate. CL occurs when the aminophthalate decays to the ground state (Xiao et al., 2002).

The effect of dissolved oxygen on the chemiluminescence of luminol on the cathode is shown in Fig.2a. Without bubbling oxygen, weak and continual luminescence exits during the process of electrolysis. The phenomenon may be because of the existence of reactive species, such as, hydrogen peroxide, superoxide, and the hydroxyl radical, which are generated by a rather low concentration of dissolved oxygen reacting on the cathode. There is bright luminescence when oxygen is bubbling to the cathode chamber, because more reactive species generated from the high concentration of oxygen dissolved in the solution. The luminescence decays within $10 \mathrm{~s}$ mainly because the low concentration $4 \times 10^{-9} \mathrm{~mol} / \mathrm{L}$ luminol may be consumed by reactive species.

Luminol univalently oxidized form reacts with $\mathrm{O}_{2}{ }^{\cdot-}$, and other radicals or compounds (e.g., $\mathrm{H}_{2} \mathrm{O}_{2}, \cdot \mathrm{OH}$ ) can also lead to luminol CL, so luminol is not a specific indicator for radicals (Lu et al., 2006; Faulkner and Fridovich, 1993). To further confirm the existence of the superoxide anion radical, 2-methyl-6-(4-methoxyphenyl)3,7-dihydroimidazo[1,2-a]pyrazin-3-one (MCLA) is used, because its CL occurs in the reaction with the superoxide ion radical and singlet molecular oxygen, but the hydrogen peroxide and hydroxyl radicals are not able to elicit the CL emission. The oxidation of MCLA with $\mathrm{O}_{2}{ }^{--}$and ${ }^{1} \mathrm{O}_{2}$ is known to lead to a highly dioxetanone intermediate, which undergoes scission to yield a light-emitting excited species, which emits luminescence (Wergifosse et al., 2004).

The chemiluminescence phenomena of MCLA were similar to luminol on cathode, according to Fig.2b. The CL intensity with bubbling oxygen was about four times that of the intensity without it. These results further affirmed the existence of a reactive species on the cathode, especially the possible generation of a superoxide radical.

However, there was no weak chemiluminescence on the anode under the same condition, which might be explained by no radical generation during electrolysis. To confirm the generation and types of radicals on the cathode, further experiments were conducted.

\subsection{Confirmation of radicals on cathode area by UV-Vis absorption spectra}

The reaction of the superoxide anion radical with NBT has frequently been used for detecting $\mathrm{O}_{2}{ }^{--}$. The superoxide anion radical produced from the chemical reactions can reduce the yellow dye (NBT) to its blue diformazan pigment, which is only slightly soluble in an aqueous solution and rapidly precipitates. The rate constant for the reduction of NBT by a superoxide is about $5 \times 10^{4}$ L/(mol·s) (Lin et al., 2001; Yamakoshi et al., 2003).

During the electrolysis procession with bubbling oxygen, the color change of NBT solution at the cathode chamber from yellow to blue was very evident, but at the anode chamber the color of the solution was from yellow to colorless. The phenomena were embodied in Fig.3. Furthermore, there was blue precipitate (diformazan) on the cathode. The above-mentioned results indicate that the NBT at the cathode chamber is reduced by a superoxide anion radical to diformazan, but at the anode chamber might be electrochemically oxidized directly (Vlyssides $e t$ al., 2004).

RNO has been reported to have specific reaction with hydroxyl radicals, as neither singlet oxygen nor various "peroxo" compounds destroy the chromophoric group of RNO (Kraljic and Trumbore, 1965). Other advantages to use RNO for the detection of hydroxyl radicals is the high rate of its reaction with $\cdot \mathrm{OH}$ radicals $\left(k=1.2 \times 10^{10}\right.$
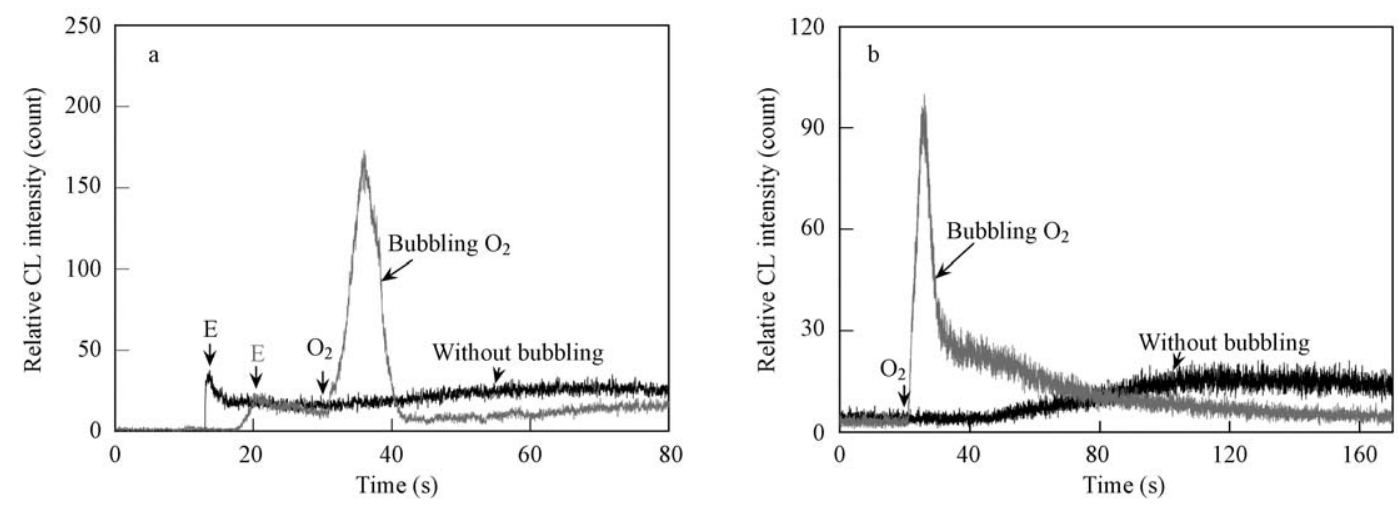

Fig. 2 Chemiluminescence (CL) of luminol (a) and MCLA (b) on the cathode. Conditions: voltage 9.00 V; current about 30 mA; luminol or MCLA concentration $4 \times 10^{-9} \mathrm{~mol} / \mathrm{L}$. E: beginning electrolysis. 

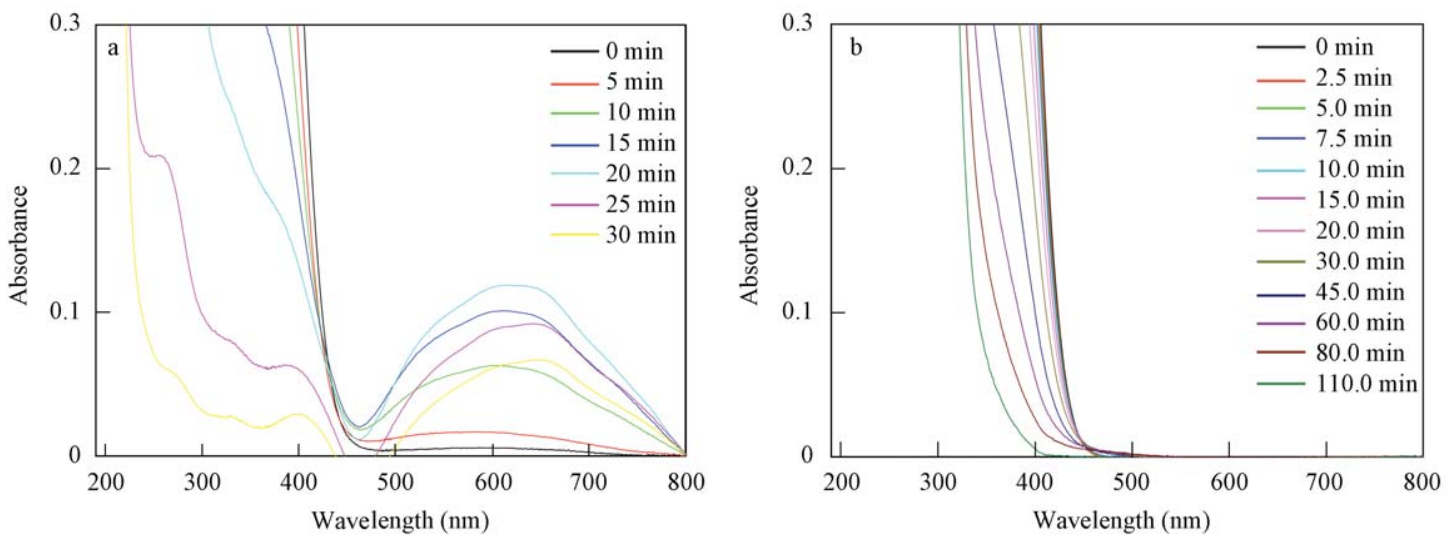

Fig. 3 UV-Vis absorption spectra change of NBT solution at the cathode chamber (a) and at the anode chamber (b). Conditions: voltage 9.00 V; current about $30 \mathrm{~mA}$; NBT concentration $10^{-4} \mathrm{~mol} / \mathrm{L}$.
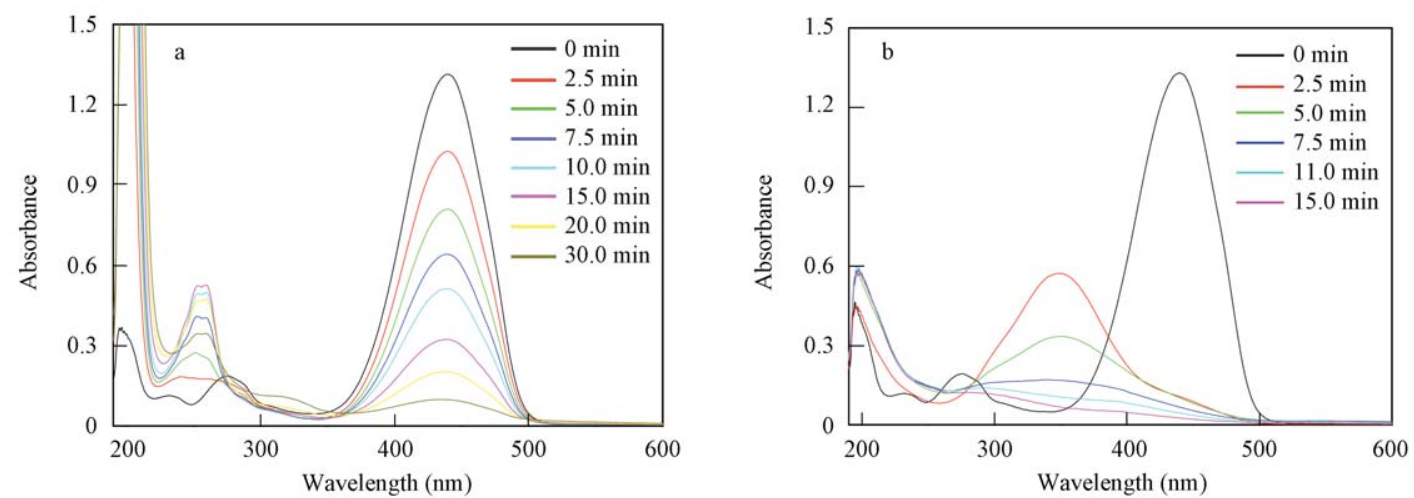

Fig. 4 UV-Vis absorption spectra change of RNO solution at the cathode chamber (a) and at the anode chamber (b). Conditions: voltage 9.00 V; current about $30 \mathrm{~mA}$; RNO concentration $2 \times 10^{-5} \mathrm{~mol} / \mathrm{L}$.

$\mathrm{L} /(\mathrm{mol} \cdot \mathrm{s})$ ) and the ease of its application, as one merely observes the bleaching of the sensitive adsorption band at $440 \mathrm{~nm}\left(\varepsilon=3.44 \times 10^{-4} \mathrm{~L} /(\mathrm{mol} \cdot \mathrm{cm})\right)($ Comninellis, 1994).

The color of the RNO solution in the two chambers turns from yellow to colorless, but their UV-Vis spectra are different from Fig.4. The decreasing adsorption band at $440 \mathrm{~nm}$ of RNO solution, in the cathode chamber, indicates the generation of a hydroxyl radical, whereas, the decoloration of RNO solution at the anode chamber may attribute to the aromatic ring opening by electrochemical oxidation, as can be seen from Fig. $4 \mathrm{~b}$, which is the same as NBT oxidation on the anode.

Without bubbling oxygen to the cathode chamber, the color of the NBT solution faded very slowly and did not turn blue. A little amount blue precipitated on the cathode surface, but it was much less than that obtained with bubbling oxygen. The color of RNO solution seemed unchanged to the naked eye, but a little bleaching of the adsorption band at $440 \mathrm{~nm}$. These phenomena might be on account of the generation of small amounts of superoxide anions and hydroxyl radicals obtained by the low concentration of dissolved oxygen. The generation rate of reactive species without bubbling oxygen is much slower than with it. Therefore, the oxygen and reactive species generation from it plays a very important role in the color change of NBT and RNO.

\subsection{Proposed mechanism of the radical generation and transformation on the cathode}

There are possibly additional steps involved in Reactions (2) and (3) during ORR according to the results of the experiments. The existence of superoxide anion proved by the formation of blue NBT diformazan and the chemiluminescence phenomena of luminol and MCLA testify that Reaction (2) may consist of the follow reactions:

$\mathrm{O}_{2}+\mathrm{e}^{-} \longrightarrow \mathrm{O}_{2}^{\cdot-}$

$\mathrm{O}_{2}^{\cdot-}+2 \mathrm{H}^{+}+\mathrm{e}^{-} \longrightarrow \mathrm{H}_{2} \mathrm{O}_{2}$

And dismutation of the superoxide anion can also form hydrogen peroxide by:

$2 \mathrm{O}_{2}{ }^{--}+2 \mathrm{H}^{+} \longrightarrow{ }^{1} \mathrm{O}_{2}+\mathrm{H}_{2} \mathrm{O}_{2}$

Shao et al. (2006) also detected the superoxide anion in the ORR on a Pt thin film electrode captured by surfaceenhanced infrared reflection absorption spectroscopy with attenuated total reflection, but the method comes with a much more complex and expensive instrument than those used in the CL and UV-Vis spectra methods.

The superoxide anion is able to oxidize many organic compounds via a complicated deprotonation-oxidation mechanism. The superoxide ion can react with a proton and a proton donor to form a perhydroxyl radical. It is also 
significant that the superoxide ion is a superonucleophile and therefore can be an origin of destructive processes initiated by the splitting off of various $\mathrm{C}-\mathrm{O}$ and $\mathrm{C}-\mathrm{Hal}$ bonds. Alkyl halides, sulphonates, and phosphates are readily involved in Reaction (7), whereas, esters, acyl halides, and acyl anhydrides react with superoxide ions by Reaction (8) (Afans'ev, 1989; Sawyer, 1991).

$\mathrm{O}_{2}^{\cdot-}+\mathrm{AX} \longrightarrow \mathrm{AO}_{2} \cdot+\mathrm{X}^{-}$

$\mathrm{O}_{2}{ }^{--}+\mathrm{RCOX} \longrightarrow \mathrm{RC}(\mathrm{O}) \mathrm{O}_{2} \cdot+\mathrm{X}^{-}$

The existence of hydroxyl radical proved by UV-Vis spectra change of RNO solution in cathode chamber and luminol CL also proves that the Reaction (3) might include the following two steps:

$\mathrm{H}_{2} \mathrm{O}_{2}+\mathrm{e}^{-} \longrightarrow \cdot \mathrm{OH}+\mathrm{OH}^{-}$
$\cdot \mathrm{OH}+\mathrm{e}^{-}+\mathrm{H}^{+} \longrightarrow \mathrm{H}_{2} \mathrm{O}$

Yu et al. (2006) also determined the hydroxyl radical in the cathode compartment of the diaphragm cell, by ESR spectrum, but the method was much more unaccessible and insensitive than the CL and UV-Vis spectra methods used in radical detection.

The most powerful oxidizing species after fluorine (3.03 $\mathrm{V}$ vs. the normal hydrogen electrode) is in fact the hydroxyl radical (2.80 V vs. the normal hydrogen electrode). The hydroxyl radical is a short-lived, extremely potent oxidizing agent, capable of oxidizing organic compounds mostly by hydrogen abstraction (Reaction (11)).

$\cdot \mathrm{OH}+\mathrm{RH} \longrightarrow \mathrm{H}_{2} \mathrm{O}+\mathrm{R} \cdot$

This reaction generates organic radicals by the addition of molecular oxygen yield peroxyl radicals (Reaction (12)). These intermediates initiate chain reactions of oxidative degradation, finally leading to carbon dioxide, water, and inorganic salts (Pera-Titus et al., 2004; Legrini et al., 1993).

$\mathrm{O}_{2}+\mathrm{R} \cdot \longrightarrow \mathrm{RO}_{2} \cdot \longrightarrow \longrightarrow \mathrm{H}_{2} \mathrm{O}+\mathrm{CO}_{2}$

The simultaneous production of evolved hydrogen at the cathode, as a byproduct (Reaction (13)), along with high power requirements, is the main aspect that should be considered.

$\mathrm{H}^{+}+\mathrm{e}^{-} \longrightarrow \mathrm{H}_{2}$

A material with high hydrogen evolution potential, such as, graphite (Do and Yeh, 1996) and mercury (Oturan and Pinson, 1995), would be an ideal cathode to improve the current efficiency. Since the number of active sites and the size of the surface area are also important factors to generate reactive species, cathodes such as reticulated vitreous carbon (Alverez-Gallegos and Pletcher, 1999) and gas diffusion electrodes (Harrington and Pletcher, 1999) were developed, to degrade organic pollution by indirect oxidation.

Superoxide anion and the hydroperoxide ion $\left(\mathrm{HO}_{2}^{-}\right)$, the conjugated base of hydrogen peroxide $(\mathrm{pKa}=11.62$ at $25^{\circ} \mathrm{C}$ ), may reach the anode and undergo a reverse reaction of 6,8 , and 9 on the cathode in an undivided cell, which consumes a lot of energy and greatly decreases the electrolysis efficiency, as the superoxide anion and hydroperoxide ion also compete with the organics for anodic reactions (Brillas et al., 2003; Kornienko et al., 2004). To avoid this disadvantage, many types of diaphragms, such as, glass frit (Do and Yeh, 1996; Harrington and Pletcher, 1999) and cationic exchange member (Kornienko et al., 2004), were used to separate two-chamber electrolysis reactors, which could reduce the degradation time and increase the total current efficiency.

The mechanism of radical generation on the cathode mentioned earlier may be useful for the development of new cathode material and an efficient reactor that generates more oxidizing radicals, to mineralize organic compounds. Furthermore, the reactive species that will be generated on the new cathode and in the new reactor may be quantified by the method of CL and UV-Vis spectra for their high sensitivity, availability, and convenience. These radical analysis methods will be the prospects for application in researching radicals in other advanced oxidation processes.

\section{Conclusions}

The generation and transformation of radicals of indirect electrochemical oxidation were confirmed by instantaneous and sensitive chemiluminescence and UV-Vis spectra. On the basis of the experiment results and relative references, mechanisms of radical generation and transformation were put forward and their future applications in indirect electrochemical oxidation were also discussed. These radical determination methods might be effective means that would help in the research of reactive species generated during other advanced oxidation processes.

\section{Acknowledgements}

This work was supported by the National Natural Science Foundation of China (No. 20437020) and the Major Research Program of the Chinese Academy of Sciences (No. YZ0632).

\section{References}

Afans'ev I B, 1989. Superoxide Ion: Chemistry and Biological Implications. Florida: CRC Press. Vol 1: 41-156.

Alverez-Gallegos A, Pletcher D, 1999. The removal of low level organics via hydrogen peroxide formed in a reticulated vitreous carbon cathode cell. Part 2: The removal of phenols and related compounds from aqueous effluents. Electrochim Acta, 44: 2483-2492.

Andreozzi R, Caprio V, Insola A, Marotta R, 1999. Advanced oxidation processes (AOP) for water purification and recovery. Catal Today, 53: 51-59.

Boye B, Dieng M M, Brillas E, 2000. Degradation of herbicide 4-chlorophenoxyacetic acid by advanced electrochemical oxidation methods. Environ Sci Technol, 36: 3030-3035.

Brillas E, Bastida R M, Llosa E, 1995. Electrochemical destruction of aniline and 4-chloroaniline for wastewater treatment using a carbon-PTFE $\mathrm{O}_{2}$-fed cathode. $J$ Electrochem Soc, 
142: $1733-1741$.

Brillas E, Calpe J C, Casado J, 2003. Mineralization of 2,4-D by advanced electrochemical oxidation processes. Water Res, 34: 2253-2262.

Comninellis C, 1994. Electrocatalysis in the electrochemical conversion/combustion of organic pollutants for waste water treatment. Electrochim Acta, 39: 1857-1962.

Do J S, Yeh W C, 1996. Paired electrooxidative degradation of phenol with in situ electrogenerated hydrogen peroxide and hypochlorite. J Appl Electrochem, 26: 673-678.

Esplugas S, Giménez J, Contreras S, Pascual E, Rodríguez M, 2002. Comparison of different advanced oxidation processes for phenol degradation. Water Res, 36: 1034-1042.

Faulkner K, Fridovich I, 1993. Luminol and lucigenin as detectors for $\mathrm{O}_{2}{ }^{-}$. Free Radic Biol Med, 15: 447-451.

Glaze W H, Kang J W, Chapin D H, 1987. The chemistry of water treatment processes involving ozone, hydrogen peroxide and ultraviolet radiation. Ozone Sci Eng, 9: 335-352.

Harrington T, Pletcher D, 1999. The removal of low levels of organics from aqueous solutions using $\mathrm{Fe}$ (II) and hydrogen peroxide formed in situ at gas diffusion electrodes. J Electrochem Soc, 146: 2983-2989.

Hirakawa T, Kominami H, Ohtani B, Nosaka Y, 2001. Mechanism of photocatalytic production of active oxygens on highly crystalline $\mathrm{TiO}_{2}$ particles by means of chemiluminescent probing and ESR spectroscopy. $J$ Phys Chem B, 105: 6993-6999.

Kornienko G V, Chaenko N V, Vasil'eva I S, Kornienko V $\mathrm{L}$, 2004. Indirect electrooxidation of organic substrates by hydrogen peroxide generated in oxygen gas-diffusion electrode. Russ J Electrochem, 40: 175-179.

Kraljic I, Trumbore C N, 1965. p-Nitrosodimethylaniline as an $\mathrm{OH}$ radical scavenger in radiation chemistry. $J$ Am Chem Soc, 87: 2547-2550.

Legrini O, Oliveros E, Braun A M, 1993. Photochemical processes for water treatment. Chem Rev, 93: 671-698.

Lin J-M, Shan X, Hanaoka S, Yamada M, 2001. Luminol chemiluminescence in unbuffered solutions with cobalt(II)ethanolamine complex immobilized on resin as catalyst and its application to analysis. Anal Chem, 73: 5043-5051.

Lu C, Song G, Lin J-M, 2006. Reactive oxygen species and their chemiluminescence-detection methods. Trac-Trends Anal Chem, 25: 985-995.

Maeda H, Yamamoto K, Nomura Y, Kohno I, Hafsi L, Ueda N et $a l ., 2005$. A design of fluorescent probes for superoxide based on a nonredox mechanism. J Am Chem Soc, 127: 68-69.

Oturan M A, Pinson J, 1995. Hydroxylation by electrochemically generated $\cdot \mathrm{OH}$ radicals. Mono- and poly-hydroxylation of benzoic acid: products and isomers' distribution. $J$ Phys Chem, 99: 13948-13954.

Panizza M, Cerisola G, 2004. Electrochemical oxidation as a final treatment of synthetic tannery wastewater. Environ Sci Technol, 38: 5470-5475.

Pera-Titus M, García-Molina V, Baños M A, Giménez J, Esplugas S, 2004. Degradation of chlorophenols by means of advanced oxidation processes: A general review. Appl Catal B-Environ, 47: 219-256.

Sawyer D T, 1991. Oxygen Chemistry. Oxford: Oxford University Press. 160-187.

Shao M H, Liu P, Adzic R R, 2006. Superoxide anion is the intermediate in the oxygen reduction reaction on platinum electrodes. J Am Chem Soc, 128: 7408-7409.

Shen Z, Yang J, Hu X, Lei Y, Ji X, Jia J, Wang W, 2005. Dual electrodes oxidation of dye wastewater with gas diffusion cathode. Environ Sci Technol, 39: 1819-1826.

Vlyssides A, Barampouti E M, Mai S, Arapoglou D, Kotronarou A, 2004. Degradation of methylparathion in aqueous solution by electrochemical oxidation. Environ Sci Technol, 38 : 6125-6131.

Wergifosse B D, Dubuission M, Marchand-Brynaert J, Trouet A, Rees J F, 2004. Coelenterazine: A two-stage antioxidant in lipid micelles. Free Radic Biol Med, 36: 278-287.

Wu Z, Zhou M, 2001. Partial degradation of phenol by advanced electrochemical oxidation process. Environ Sci Technol, 35: 2698-2703.

Xiao C, Palmer D A, Wesolowski D J, Lovitz S B, King D W, 2002. Carbon dioxide effects on luminol and 1,10-phenanthroline chemiluminescence. Anal Chem, 74: 2210-2216.

Xu X, Wang W, Li H, 1998. Determination of hydroxyl radicals in Fenton reaction by chemiluminescent method and its application. Environ Sci, 19: 51-54.

Yamakoshi Y, Umezawa N, Ryu A, Arakane K, Miyata N, Goda Y, Masumizu T, Nagano T, 2003. Active oxygen species generated from photoexcited fullerene (C60) as potential medicines: $\mathrm{O}_{2}{ }^{\cdot-}$ versus ${ }^{1} \mathrm{O}_{2}$. J Am Chem Soc, 125: 1280312809.

Yu X J, Wang H, Sun D Z, Song L W, Wu L, 2006. Mechanism study of electrochemical oxidation in the terylene diaphragm cell. J Environ Sci, 18(1): 33-39. 